respiratory activity that is perhaps analogous to complete heart block. The only way at present to overcome this problem seems to be by the retention of some part of the host's intact lung to "drive" the graft. Secondly, after transplantation there is a perplexing loss of function at capillary-alveolar level resulting in an early deficiency of ventilation and oxygen uptake in the graft. Though this may improve, function tends to remain well below normal. ${ }^{3}$ To some extent the loss can be reduced in the experimental animal by increasing the functional load on the transplant by further excision of the remaining normal lung tissue of the recipient. This is a state of affairs of which we understand the principle from the behaviour of heart valve transplants: it seems essential that their function should not be shared. But in addition the loss of lung function may, it seems, at least in part, be due to pulmonary oedema from obstruction of the pulmonary venous drainage.

Technically transplantation of the lung, like that of most other organs, is a relatively easy operation, though it may still be improved, for example, by the addition of bronchial-artery implantation into the aorta, theoretically not a very difficult procedure. Human lung homografts have now been recorded in five patients, including the case described at page 759 of the B.M.F. this week by Dr. Henry Matthew and his colleagues at Edinburgh.

Indications for lung transplantation cannot at this stage be laid down, but it would appear that only patients who have a rapidly fatal disease should be considered as recipients, and among these the operation may have to be restricted to patients in whom a certain amount of lung remains intact. Paraquat poisoning would seem to be a good indication, provided sufficient time can be allowed to elapse for the complete elimination of the drug; otherwise the graft may be destroyed.

Finally, study of the clinical and pathological findings in patients after homograft operations suggests that much further research is needed to enable surgeons to distinguish between "inflammations" resulting from bacterial infection, chemical reaction, and rejection processes. Until these can be accurately separated the conclusions that may be drawn from individual cases will be in doubt.

Despite the paucity of long-term survivors among experimental animals and the poor record of human lung homotransplants so far, this work seems likely to continue. If it does, it should surely be taken along cautiously only in the few centres fully equipped for it, and by teams which have already gained a thorough experience of immunosuppression as at Edinburgh.

\section{A New Anticoagulant}

An entirely safe and reliable anticoagulant for the prophylaxis or treatment of thrombo-embolism has yet to be discovered, but recently an entirely new approach to the problem has been made. An enzyme which directly destroys fibrinogen has been extracted from the venom of the Malayan pit viper, Agkistrodon rhodostoma. Given intravenously, the enzyme reduces the concentration of fibrinogen in the plasma to very low levels by destroying the protein faster than it can be synthesized. The oral anticoagulants, such as warfarin or phenindione, depress the hepatic synthesis of clotting factors. Heparin directly inhibits coagulation enzymes, particularly thrombin. In contrast, the anticoagulant effect of the venom enzyme depends on the restriction of fibrinogen, the soluble precursor of fibrin.

Interest in the Malayan pit viper as the source of a therapeutic substance was prompted by $\mathrm{H}$. A. Reid, who observed that bleeding was negligible in patients bitten by the snake, despite a state of defibrination. Their blood was often incoagulable owing to a low concentration of fibrinogen, sometimes for many days. ${ }^{1}$ The active principle has now been separated from neurotoxic and vasculotoxic (haemorrhagic) fractions of the venom ${ }^{2}$ and is available for clinical trial as Arvin. Its action is independent of the coagulation and fibrinolytic enzyme systems, and platelets are not affected, in either numbers or function. It is usually assayed in units defined by its ability to clot fibrinogen.

Extensive studies in animals ${ }^{\mathbf{3}}$ and preliminary clinical trials $^{45}$ suggest that when defibrination is induced by Arvin spontaneous bleeding from normal tissues is rare and menstrual losses are not increased. Such bleeding as may occur from recent surgical wounds has been easily controlled. This contrasts favourably with the record of the conventional anticoagulants. Animal studies show that the injection of $\boldsymbol{A}$. rhodostoma venom causes the formation of intravascular microclots, which rapidly disappear. ${ }^{6}$ The fibrinolytic enzymes may help in this removal. The weak structure of the clots makes them peculiarly susceptible to lysis, and there is evidence that the macrophages of the reticuloendothelial system play a part. Toxic effects due to obstruction of small blood vessels develop only when the Arvin is given too quickly, so that the removal mechanisms are overwhelmed. The safety margin is wide ; in both mice and dogs the lethal dose is 500 to 1,000 times greater than the defibrinating dose.

Occasional hypersensitivity reactions have been reported, and dogs develop some resistance to the enzyme when it is given for long periods. However, no serious reactions have been encountered and no precipitating antibodies detected. ${ }^{5}$

Arvin therefore shows many of the characteristics of the ideal anticoagulant. It causes a predictable, reproducible, and sustained effect, easily controlled by measurement of the plasma fibrinogen or by a simple test of clot quality. ${ }^{8}$ When its administration is stopped its effect is tapered off, which makes "rebound" thrombosis unlikely. The concentration of fibrinogen in the plasma slowly rises to pretreatment levels over several days. If the need arises a rapid return to normal can be achieved by giving a specific antivenom, prepared in horses, and a transfusion of fibrinogen.

Preliminary clinical studies ${ }^{4}$ have indicated some of the benefits which can be achieved in the treatment of arterial or venous thrombosis. Controlled clinical trials should not be long delayed.

Reid, H A. Thcan, P. C. Chan, K. E., and Baharom, A. R., Lancet, $1963,1,617$.

Esnouf, M. P., and Tunnah, G. W., Brit. F. Haemat., 1967, 13, 581.

3 Ashfcrd, A., Ross, J. W., and Southgate, P., Lancet, 1968, 1, 486.

- Bell, W. R., Pitney, W. R., and Goodwin, J. F., Lancet, 1968, 1, 490.

- Sharp, A. A., Warren, B. A., Paxton, A. M., and Allington, M. J., Lancet, 1968, 1, 493.

- Regoeczi, E., Gergely, J., and McFarlane, A. S., \}. clin. Invest., 1966, 45, 1202.

'Reid, H. A., in Anımal Toxins, ed. F. E. Russell and P. R. Saunders, 1967, p. 323 . Oxford.

* Rcid, H. A., and Chan, K. E., Lancet, 1968, 1, 485. 